\title{
Deafness-hypogonadism syndrome
}

INSERM

\section{Source}

INSERM. (1999). Orphanet: an online rare disease and orphan drug data base. Deafnesshypogonadism syndrome. ORPHA:90646

This syndrome is characterized by the association of congenital mixed hearing loss with perilymphatic gusher (Gusher syndrome or DFN3; see this term), hypogonadism and abnormal behavior. 